Waste and Resource Management Volume 164 Issue WR2

Resolution of electrical imaging of fluid movement in landfills

Jolly, Beaven and Barker
Proceedings of the Institution of Civil Engineers

Waste and Resource Management 164

May 2011 Issue WR2

Pages 79-96 doi: 10.1680/warm.2011.164.2.79

Paper 1000037

Received 29/10/2010

Accepted 22/02/2011

Keywords: landfill/research \& development/waste

management \& disposal

ICE Publishing: All rights reserved

\title{
Resolution of electrical imaging of fluid movement in landfills
}

1 Joanna M. Jolly (née Moore) MSc, PhD, MCIWEM Earth Sciences, University of Birmingham, Edgbaston, Birmingham, UK now at Environment Agency, Birmingham, UK

2 Richard P. Beaven MSc, PhD, FGS

Principal Research Fellow, Waste Management Research Group, School of Civil Engineering and the Environment, University of Southampton, Southampton, UK
3 Ron D. Barker MSc, PhD

Honorary Lecturer in Environmental Geophysics, School of Geography, Earth and Environmental Sciences, University of Birmingham, Edgbaston, Birmingham, UK
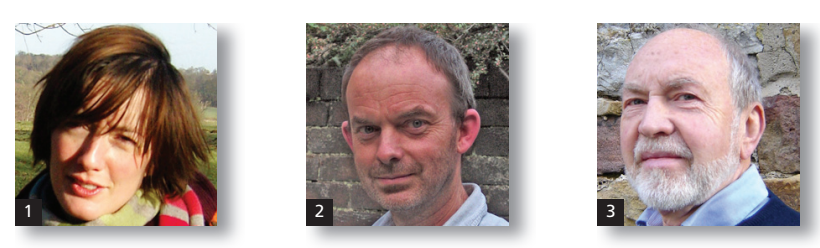

This paper investigates the efficacy of time-lapse electrical imaging using surface electrodes to monitor the movement of leachate within a landfill. A laboratory-based study allowed direct comparison between observed physical dewatering and time-lapse electrical imaging data. In combination with a forward modelling study this demonstrated the limitations and advantages of this geophysical technique in terms of its applicability, resolution and complementarity to conventional dewatering monitoring techniques. It also confirmed the nature, likely magnitude and impact of artefacts created by the resistivity inversion process and highlighted the potential for misinterpretation of results. An 18-month study provided field results comparing hydrogeological and imaging data during the dewatering of a real landfill system. Insights gained from the laboratory study and forward modelling exercise greatly enhanced the interpretation of the field data, enabling the method to be applied with greater confidence in the future. The need to combine a forward modelling exercise with any interpretation of resistivity data is clearly demonstrated.

\section{Introduction}

In response to stricter legislation governing the operation and monitoring of landfills, and with progressively more emphasis on the controlled biodegradation of waste, a robust understanding of the physical and biochemical processes taking place within landfills is essential. Geophysical techniques already play an important role, both with the installation of below liner leak detection systems (Taylor et al., 1999), and as a tool for tracking changes in water content in landfills (e.g. Guerin et al., 2004; Rosqvist et al., 2005).

Many operators now realise the potential benefits of running landfills as controlled bioreactors, which often involves the controlled introduction or recirculation of leachate (White et al., 2011) to accelerate degradation actively and enhance landfill gas production rates. There are actually many reasons why leachate recirculation in landfills is beneficial (Benson et al., 2007; Environment Agency, 2009; Knox et al., 2007) but this increases the need to understand the way in which the recirculated leachate becomes distributed in a site. The possibility that rapid short circuiting of large volumes of leachate from the injection infrastructure to the collection system could result in a large proportion of the waste being bypassed means that there is a real need to be able to monitor the movement of leachate. Resistivity as a geophysical sensing technique has the potential to do this, and has indeed been used (Grellier et al., 2008; Guerin et al., 2004; Moreau et al., 2004; Rosqvist et al., 2003; 2005; 2007; 2011), but the advantages and limitations of the technique need to be fully understood.

This paper describes an integrated research programme that investigated the use of time-lapse electrical imaging to monitor the effect of dewatering in both controlled laboratory conditions and at a full-scale landfill over a period of 18 months together with a modelling exercise to investigate limitations in the resolution of the technique and the interpretation of results. As such it represents one of the longest studies of this type yet reported in the literature. 


\section{Time-lapse electrical imaging}

\subsection{The resistivity method}

In the resistivity method, artificially generated electrical currents are introduced into the ground through electrodes and the resulting potential differences are measured at the surface using other electrodes in the vicinity of the current flow. Quantitative information on the shape and electrical properties of subsurface heterogeneities are derived from the deviation of the measured distribution of potential differences from that expected for a homogeneous ground.

The dominant mechanism by which most geological media conduct electricity is by the passage of ions within pore waters. Therefore, the dominant factors in determining the resistivity of subsurface media is its porosity (or the presence of fluid-filled fractures and fissures) and the salinity of the fluid saturating these voids. Variations in the temperature of the saturating fluid also give rise to variations in electrical resistivity.

\subsection{Electrical imaging}

Electrical imaging describes the automated process of gathering multiple measurements of resistivity to build up either a vertical two-dimensional cross-section of data or a three-dimensional block of data. The measurements referred to in this paper are in two dimensions and the method is illustrated in Figure 1. Numerous electrode array configurations can be used in electrical imaging, with the most appropriate choice being dependent on a number of factors including: the type of structure to be mapped; the sensitivity of the resistivity meter and the background noise level; the sensitivity of the array to vertical and horizontal changes in the subsurface resistivity; the depth of investigation; the horizontal data coverage; and the signal strength.

In the work presented in this paper the Wenner-alpha array was used, as it is good at resolving changes in the vertical direction (which matches layering and the potential manner in which dewatering would occur) and has one of the strongest signal strengths, allowing a high signal-to-noise ratio to be maintained, essential when working in areas of low resistivity such as a landfill.

Typically, two multi-core cables, each containing numerous electrode connections, are positioned in a straight line along the surface and the electrodes are deployed along this line with an equal interelectrode spacing, $a$. Once a measurement has been determined for one set of four electrodes, the next set of four electrodes along the line is automatically selected. Once the end of the line is reached, the survey is repeated but with an electrode spacing of $2 a$ and so on, up to a maximum number dictated by the number of electrodes used. The wider the electrode spacing, the greater the volume of the subsurface sampled, which in turn represents a deeper measurement, as plotted on the cross-section of measurements shown in Figure 1. The measured apparent resistivity values are plotted at the midpoint of the four electrodes used to acquire them and at a depth of approximately half the interelectrode spacing, to form what is termed a 'pseudosection', a two-dimensional vertical cross-section of data points.

Although the pseudosection acquired from the electrical imaging method can be viewed as an approximate representation
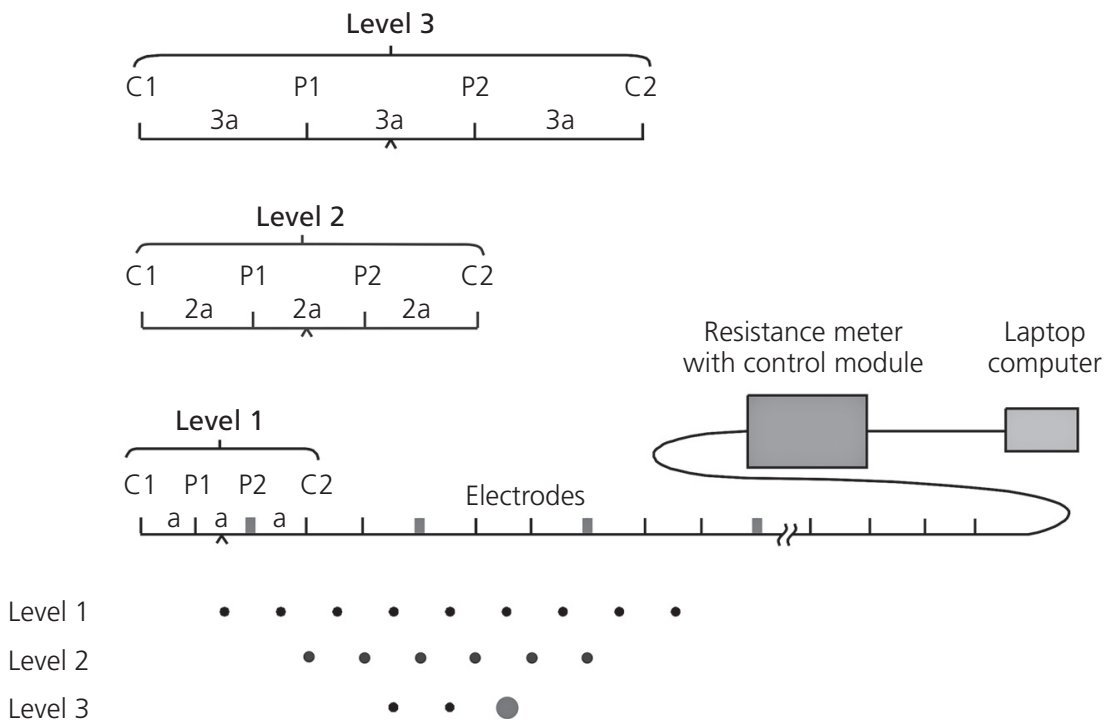

Figure 1. Schematic representation of the electrical imaging technique using the Wenner-alpha electrode array 
of the subsurface and indeed contains information on the subsurface geology, it is also influenced by the electrode geometry. In order to separate geometrical effects and produce an image of true, or model, formation resistivities and depths, the pseudosection is put through a processing stage called 'inversion'. Inversion is a means of estimating the 'cause of an effect'. The inversion routine used in this research is RES2DINV, based on the technique of Loke and Barker (1996).

Although the two-dimensional model obtained from the inversion scheme gives an estimate of the true subsurface resistivity distribution, this model is not unique: many geological models could give rise to the same apparent resistivity pseudosection. In one-dimensional inversion, the principle of equivalence states that the sounding curve [a log-log plot of apparent resistivity $\mathrm{Ohm}$-metres $(\Omega \mathrm{m})$ against electrode spacing $(\mathrm{m})]$ due to a thin high resistivity layer is indistinguishable (within the limits of the measurement errors) from that due to a thicker layer with a lower resistivity. It is therefore desirable to have some ground truth data to constrain the inversion process. This may take the form of depth to electrical interfaces (e.g. the base of a landfill site) or values of true resistivity acquired from borehole logging.

It is also important to appreciate that the inversion process may generate a model that shows unrealistic variations in the model resistivity values and may not be characteristic of geological features, so care needs to be taken in the interpretation of electrical imaging data. Signal noise, topography and out-of-section three-dimensional effects can all influence results. Furthermore, it must be remembered that the graphical representation of the results as contour maps has been undertaken by computer packages that smooth out a grid of data.

\subsection{Application to the study of fluid movement}

The primary limitation to most hydrogeological monitoring techniques is a lack of spatial resolution describing subsurface heterogeneity. However, hydrogeological methods do produce ground truth data, which are unequivocal in terms of the fluid composition or the height of the water table at the point of measurement. Conversely, the primary strength of geophysical methods is the ability to quantify the spatial variability of subsurface physical properties, although its main drawback is the ambiguity inherent in any interpretation. Consequently, it is apparent that the dual use of hydrogeological monitoring and geophysical techniques can be mutually beneficial and can result in a more greatly enhanced understanding of site conditions than could be obtained from either method alone.

A method to improve the inherent ambiguity in geophysical interpretation is to observe only the change in geophysical properties of the system over time. This form of monitoring is termed 'time-lapse', and was the primary method used in this study.
Time-lapse electrical imaging can be extremely powerful in its ability to provide continuous spatial information on changes in physical properties in the subsurface. In terms of electrical properties, these changes can be caused by a change in conductivity of the fluid in the pore spaces, a change in the saturation of the pore spaces or a change in temperature of the subsurface.

\section{Laboratory study}

One of the main problems with time-lapse electrical imaging is that interpretation of these studies remains qualitative and often difficult to validate. Consequently, in an attempt to validate the interpretation of electrical resistivity measurements recorded during subsurface changes in saturation, a laboratory experiment was undertaken to simulate a groundwater pumping test in a porous medium aquifer with concurrent measurements of electrical resistivity. The purpose of this study was to test the resolution of the electrical imaging technique on a known subsurface with controlled hydraulic conditions. It is only in this way that the resolution of the technique could be fully assessed and understood. It was considered essential to establish this level of understanding before the interpretation of a more complex environment, such as a landfill, could be attempted.

Initially, the tank was filled with a well-sorted sand to represent a homogeneous model. A body of contrasting hydraulic properties was then implanted to observe the effects of heterogeneities during dewatering. Full details of the study are provided by Moore (2004).

\subsection{Experimental design}

The dewatering experiments were undertaken in a transparent 'thin-slice' tank which was $3 \mathrm{~m}$ long, $65 \mathrm{~cm}$ high and $9 \mathrm{~cm}$ wide (Figure 2). The tank was filled with a medium-grained $(0.5-1.0 \mathrm{~mm})$ washed sand comprising over $99 \%$ quartz chosen to provide a moderate capillary fringe height of approximately $9 \mathrm{~cm}$. Following the careful and even placement of sand, the tank was filled with water coloured with fluorescein dye from the base upwards to drive out air from the pore spaces and minimise the formation of trapped air pockets.

Constant heads were maintained at each end of the tank by pumping into two fully penetrating slotted $5 \mathrm{~cm}$ diameter pipes covered with geotextile wrap. The tank was dewatered by allowing gravity drainage to occur through a central borehole of $2 \mathrm{~cm}$ diameter, slotted over its full length and again covered in geotextile wrap. The discharged water was then recirculated to supply the fixed heads, using a centrifugal pump and gate valves to limit the flow rate. Ten observation wells were positioned approximately symmetrically about the central drainage well as shown in Figure 2.

The experiment was instrumented with 50 stainless steel electrodes positioned at the surface of the sand with an 


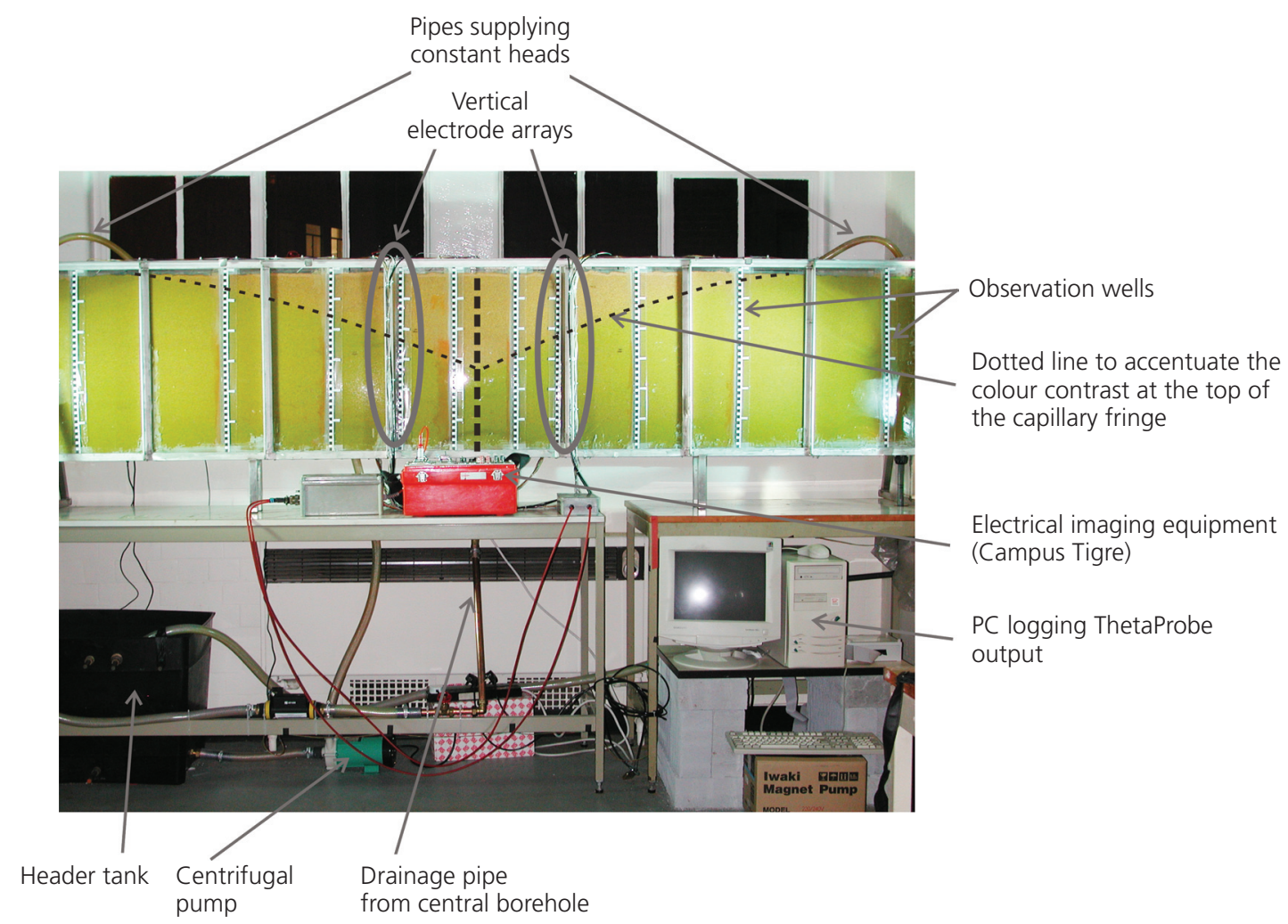

Figure 2. Annotated photograph of the laboratory tank drainage experiment. For colour versions of figures please see www. icevirtuallibrary.com/content/article/10.1680/warm.2011.164.2.79

interelectrode spacing of $5.5 \mathrm{~cm}$. Each electrode was a line source (i.e. extends across the full width of the tank) and extended to a depth of $2.5 \mathrm{~cm}$ into the sand.

Measurements of resistivity were carried out using a Campus Tigre (Allied Associates Geophysical Ltd) A total of 392 measurements was recorded in each image using a Wenneralpha array (Figure 1). The currents used ranged from $0.5 \mathrm{~mA}$ at the smaller electrode spacings to $5 \mathrm{~mA}$ at the larger electrode spacings to maintain signal strength. The current-on time was $1 \mathrm{~s}$ and the off time $0.5 \mathrm{~s}$. Each measurement of resistivity was repeated three times and the repeatability quantified in terms of a percentage error. Errors were always less than $1 \%$.

To ascertain the variation of resistivity with saturation through the depth of the tank, two vertical arrays of electrodes were also installed $32 \mathrm{~cm}$ and $37 \mathrm{~cm}$ from the central drainage well. Each array contained 25 electrodes with a $2 \mathrm{~cm}$ interelectrode spacing. The electrodes were stainless steel rods $1.5 \mathrm{~cm}$ long.

Four ThetaProbes (type ML2x; Delta-T Devices), to provide a direct measurement of moisture content, were also installed in close proximity to the vertical electrode arrays at depths of 17 and $25 \mathrm{~cm}$. Before the pumping test simulation, a separate drainage experiment was carried out in the tank to provide data that allowed a relationship to be determined between changes in water content (as measured by the ThetaProbes) and change in resistivity (as measured by the vertical electrode arrays). The calibration curve is given in Figure 3. Further technical details of the experiment, including a description of potential errors (e.g. from the large surface area of the electrodes) and calibration and correction techniques employed, are provided by Moore (2004).

\subsection{Dewatering experiment}

The pumping test simulation took place as a series of four steady-state tests (Homl to Hom4), in which the discharge rate from the central well was increased incrementally (Figure 4), thereby creating larger drawdowns around the well. A resistivity profile was taken before the start (i.e. at full saturation) and then at each dewatering stage. Time $(1.5 \mathrm{~h}$ was determined to be sufficient) was allowed at each step for hydraulic equilibrium to be reached before electrical imaging took place.

After correction for the depth of penetration of the electrodes (see Moore, 2004), the values of apparent resistivity (derived 


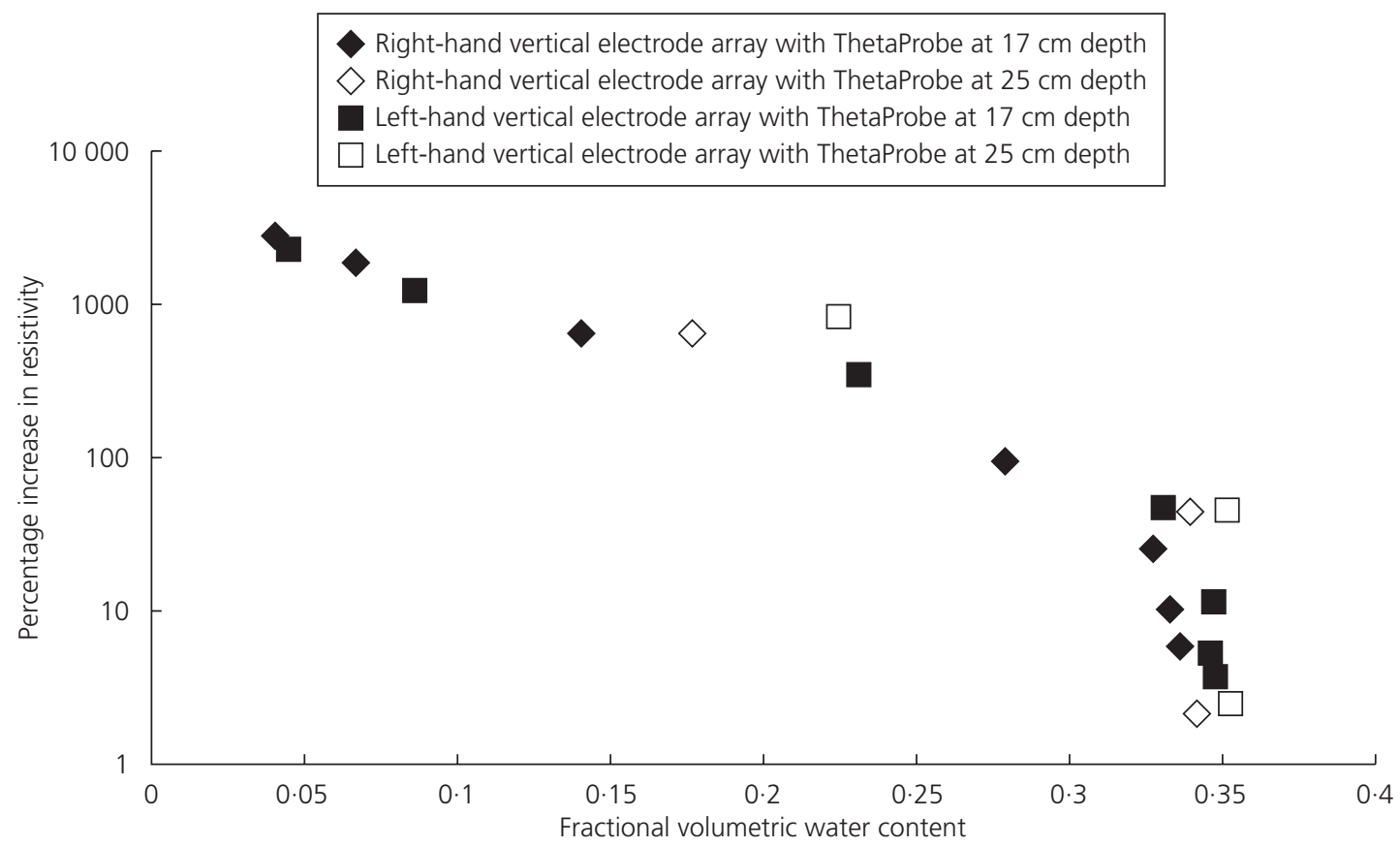

Figure 3. Percentage increase in resistivity, recorded by the vertical electrode arrays, against fractional volumetric water content, measured by the ThetaProbes

from the raw data) were processed using the inversion routine RES2DINV (Loke and Barker, 1996). To minimise ambiguity in the inversion of apparent resistivity to model resistivity, it is good practice to fix the location and/or resistivity value of any known electrical boundary. In this experiment, the tank walls form boundaries of enormous electrical contrast with the fill material and were therefore incorporated into the inversion process. Figure 4 shows the percentage changes from initial, fully saturated conditions. For comparative purposes, actual water level readings as measured in the 10 observation wells are also drawn on the cross-sections. The drainage pattern can be seen clearly by the areas of increased resistivity, relating to a decrease in saturation. The percentage increase in resistivity recorded during this drainage experiment was extremely high, reaching values of over $3000 \%$ (indicated by symbol 'a' on Figure 4). The yellow colour (b) represents a contour interval of $\pm 4 \%$ change in resistivity, effects attributable to measurement error and errors incurred in the inversion process. Apart from Homl (in which dewatering had not extended far enough below the electrode penetrations) the imaging results show very good correlation with the physical extent of the drainage, particularly in the central part of the image. The imaging at each end of the tank (c), where data are sparse, appear less well constrained and do not agree as closely with the observation well measurements. The area of decreased resistivity, shown by the green colours (d) at the base of the image does not agree with the physical processes known to be occurring within the tank (an increase in saturation is not possible) and therefore must be an artefact produced by the inversion process. Similarly, artefacts of the inversion process are seen in the areas of increased resistivity (f) on the side lobes of the images in locations that remain below the water table.

The creation of artefacts during the inversion process were described by Park (1998) and are now a well-known phenomenon within the landfill literature (Clement et al., 2010; Jolly et al., 2007; Moore, 2004) and elsewhere (Olayinka and Yaramanci, 2000). However, there have been a number of early landfill-related geophysical studies that did not necessarily discuss inversion artefacts during the interpretation of results. This is discussed further in Section 4.3.

To interpret these time-lapse images accurately, it is important to understand how resistivity varies with depth through the tank. Figure 5 shows a photograph of the capillary fringe highlighted within the sand by green fluorescein dye and extending (visually) to a height of approximately $8 \mathrm{~cm}$. The capillary fringe is the gradational change in saturation above the water table, where moisture is held in the pore spaces by capillary action. A gradational change in water content will manifest itself in a gradational change in resistivity, and this was indeed measured by one of the vertical electrode arrays when 


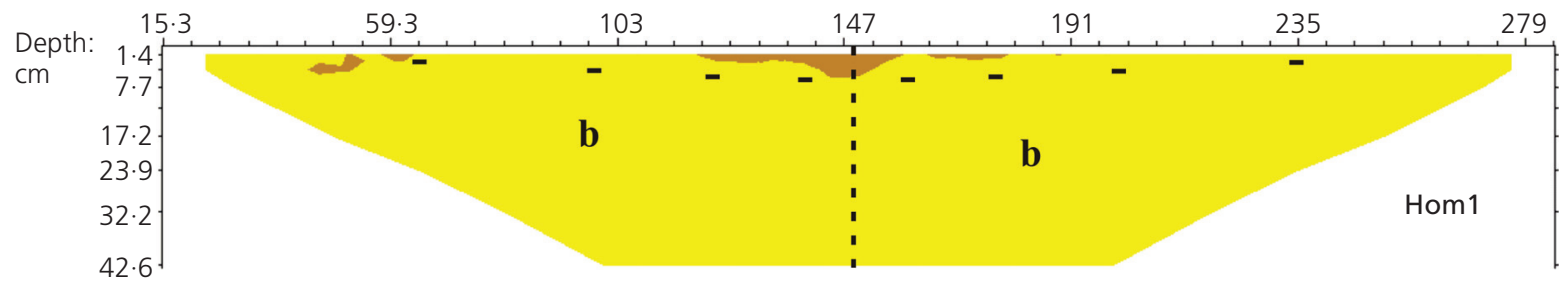

$(\mathrm{cm})$
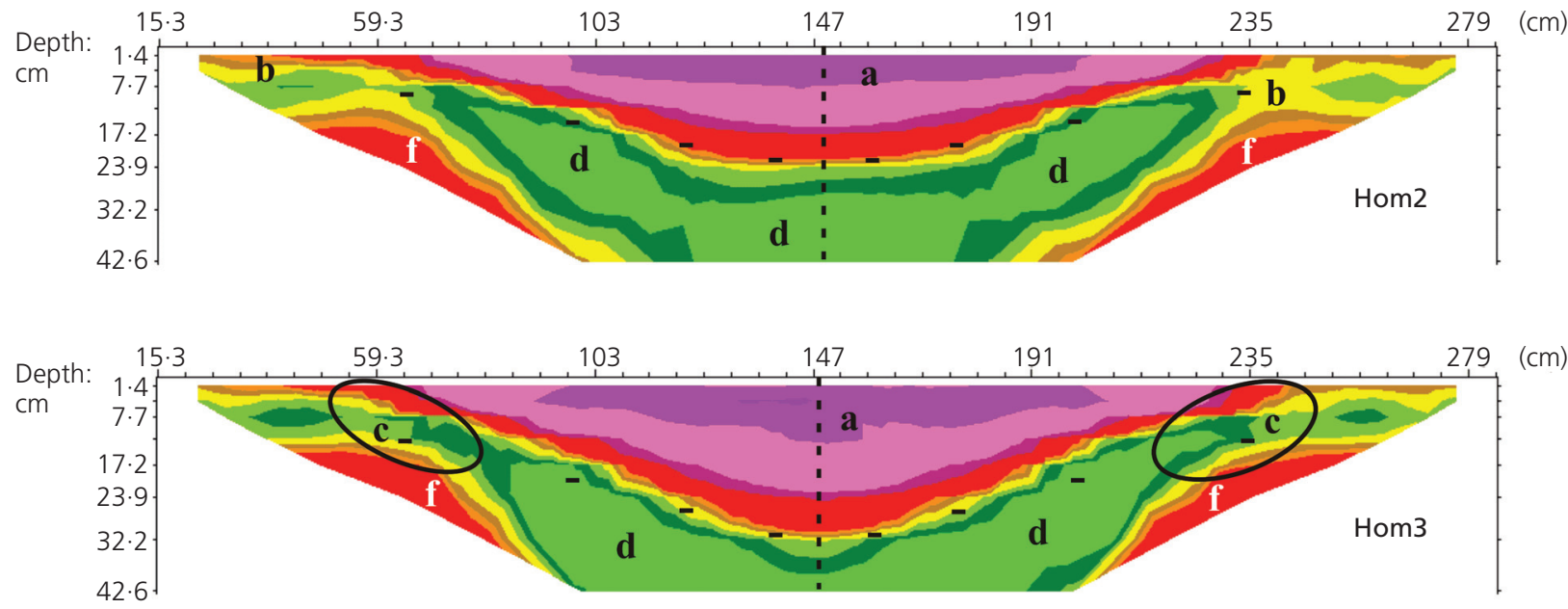

$(\mathrm{cm})$

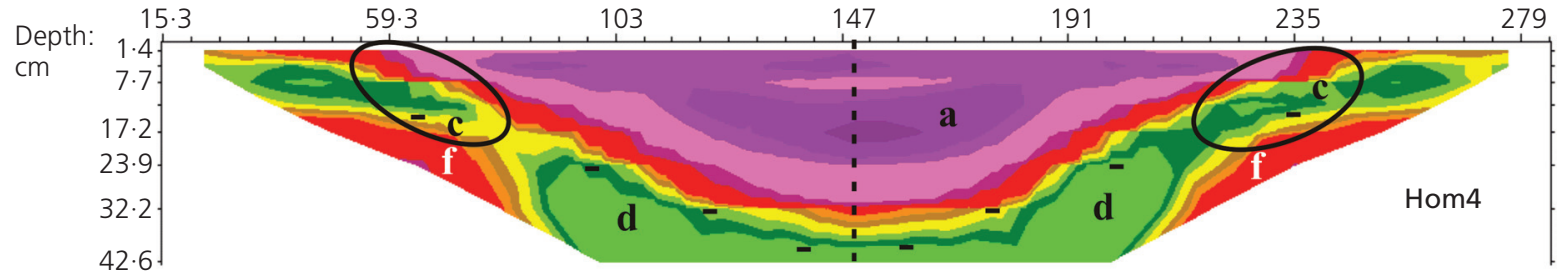

$(\mathrm{cm})$

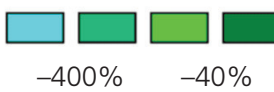

$-4 \%+4 \%$

$+40 \%$

$+400 \% \quad+4000 \%$

Figure 4. Percentage changes in resistivity recorded during a fourstage (Hom1 to Hom4) drainage exercise in a homogeneous

sand-filled tank. Water levels remained fixed at $0 \mathrm{~cm}$ depth at each end of the tank, and drainage occurred from the centre of the image $(150 \mathrm{~cm})$. The drainage can be seen by areas of increased resistivity (a), reaching a value of over $3000 \%$. The yellow colour (b) represents $\mathrm{a} \pm 4 \%$ change in resistivity, attributable to errors incurred in the measurement and inversion process. A decrease in resistivity (d) of up to $50 \%$ can be seen at the base of the images. For colour versions of figures please see www.icevirtuallibrary.com/ content/article/10.1680/warm.2011.164.2.79

the tank had been drained to a water table height of $21.5 \mathrm{~cm}$ above the base of the tank and the water table was horizontal across the tank. Figure 5 shows that the values of resistivity do not start to decrease immediately above the water table, as very little or no changes in saturation have occurred at this height. At $3.5 \mathrm{~cm}$ above the water table the values of resistivity begin to decrease and continue to decrease to a height of $13.5 \mathrm{~cm}$ above the water table - this has been interpreted as the full extent of the capillary fringe.

To translate these observations to the time-lapse imaging results, the images in Figure 4 have been re-plotted as volumetric water content in Figure 6 using the relationship shown in Figure 3. In addition to the position of the water table (as 


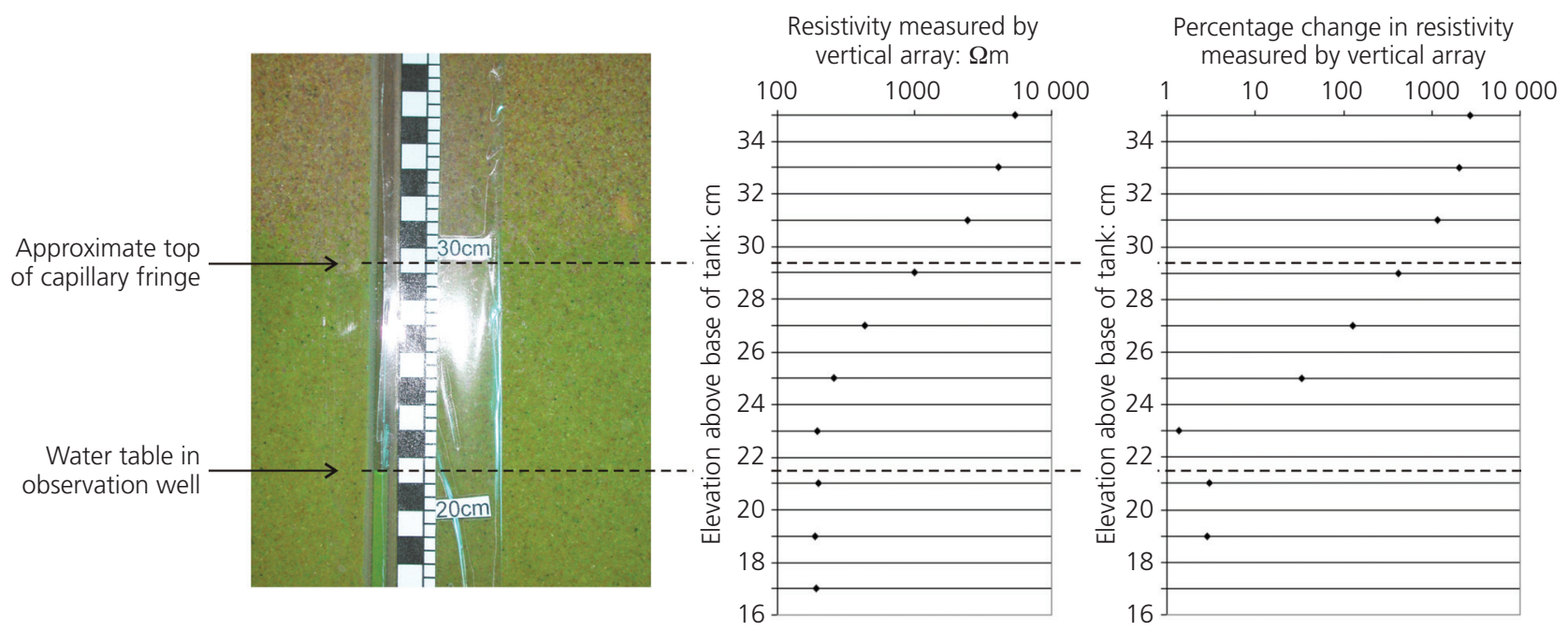

Figure 5. Photograph showing the capillary fringe supported by the sand used in the laboratory drainage experiment, highlighted by green fluorescein dye. The visual extent of the fringe reaches approximately $8 \mathrm{~cm}$ above the water table, seen at $21.5 \mathrm{~cm}$ in the observation well. The left-hand graph shows a profile of resistivity as measured by one of the vertical arrays at the same time (for a horizontal water table). The right-hand graph shows the same values of resistivity represented as percentage changes in resistivity from the value of resistivity measured at $17 \mathrm{~cm}$ above the base of the tank. For colour versions of figures please see www.

icevirtuallibrary.com/content/article/10.1680/warm.2011.164.2.79

measured in the observation wells) a height of $13.5 \mathrm{~cm}$ has been marked, indicative of the position of the capillary fringe. There is good correlation between the two sets of data, which suggests that time-lapse electrical imaging, using surface measurements of resistivity, can provide quantitative information of changing electrical conditions in the subsurface when used in conjunction with calibration data.

To test the technique of time-lapse electrical imaging in the study of heterogeneous ground, an anomaly of contrasting hydraulic properties was emplaced within the sand fill. Two types of anomaly were used in this study: absorbent paper to represent a less freely draining medium (e.g. municipal solid waste) and gravel to drain even more freely than the mediumgrained sand fill. Electrical resistivity images were recorded at full saturation and at two subsequent stages of drainage using the same technique as described above.

The percentage changes in resistivity observed for the absorbent paper anomaly are shown in Figure 7. The location of the anomaly is outlined by the black rectangle, and the observation well readings are shown by the black dashes. The time-lapse imaging results from the paper anomaly (Figure 7) show good correlation with the observation well measurements at the centre of the images but, as for the homogeneous case, the side areas deviate slightly from the drainage pattern. However, the observation well data show symmetrical changes in water level and do not indicate the presence of a hydraulic anomaly and suggest the tank fill to be homogeneous. The time-lapse results show strong asymmetry and accurately resolve the location of an anomaly less freely draining than the surrounding material.

Figure 8 shows the percentage changes in resistivity associated with the gravel anomaly, in the same location as the paper anomaly and with the observation well readings marked. The gravel anomaly is very clearly defined by the time-lapse results, particularly the top image in which the medium-grained sand supports an $8 \mathrm{~cm}$ high capillary fringe, whereas the gravel matrix has negligible capillary forces and so the electrical contrast is greatest at this level. The anomalous area is still visible in the lower image, but the electrical contrast between these two highly resistive (clay free) and freely draining media is not high at full drainage. Again, this hydraulic anomaly is not readily identifiable from the near perfectly symmetrical measurements of water level.

\section{Field study}

\subsection{Site conditions and field method}

Time-lapse electrical imaging was used to monitor the effect of dewatering a landfill site in Essex, UK, from April 2000 for a 

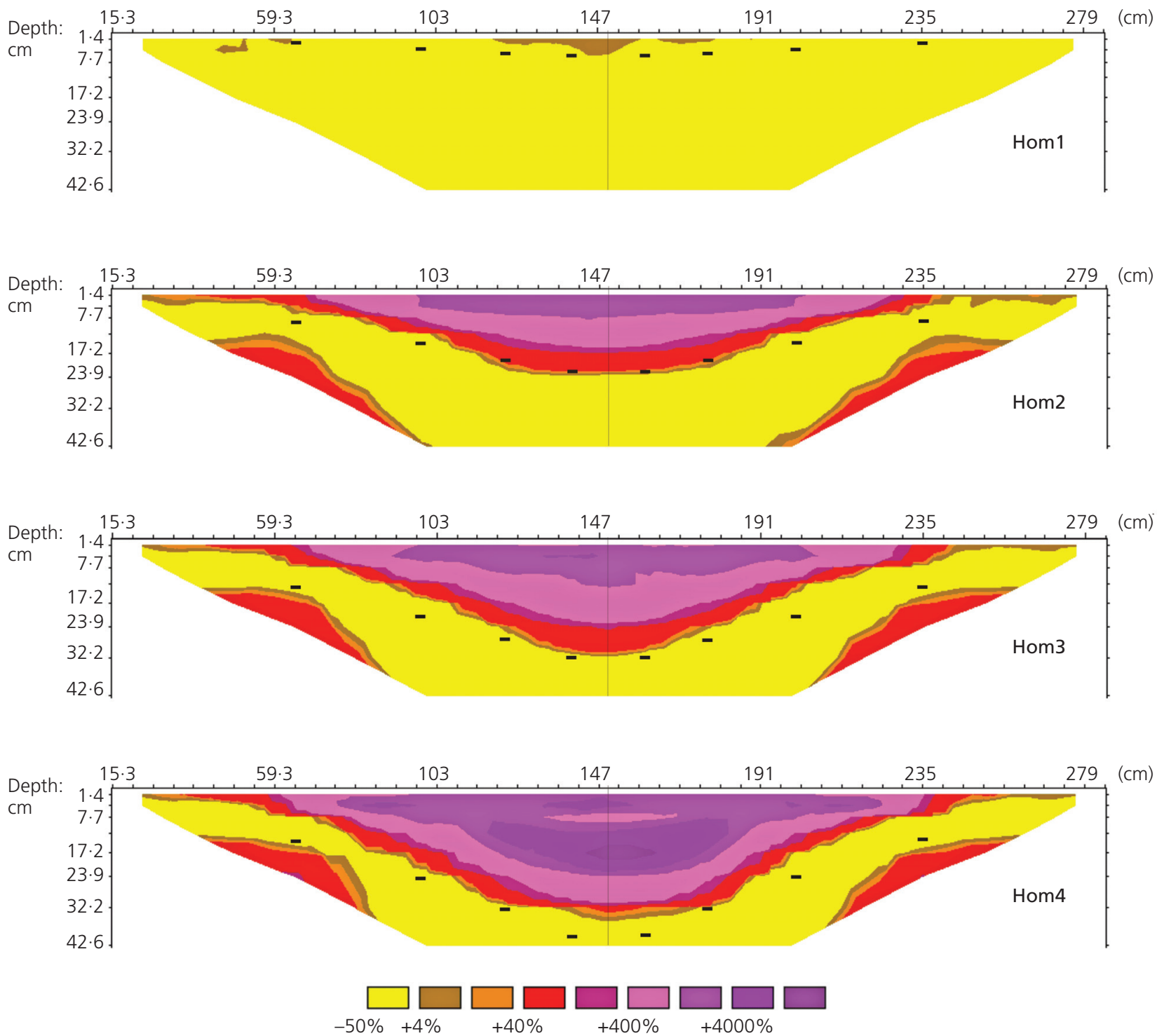

Figure 6. Time-lapse images recorded during the sand tank drainage experiment, displayed in terms of volumetric water content, as inferred from the graph shown in Figure 3. Also shown on these images is the $13.5 \mathrm{~cm}$ distance, above the position of the water table, over which the resistivity decreases by more than $+2000 \%$. This distance is determined from the measurements of resistivity recorded by the vertical electrode arrays (Figure 5) and relates to the presence of the capillary fringe. For colour versions of figures please see www.icevirtuallibrary.com/content/article/ 10.1680/warm.2011.164.2.79

period of 18 months. The landfill dewatering was achieved using three horizontal leachate drains (HW1 to HW3), each $250 \mathrm{~m}$ long and drilled to an elevation of approximately $3.5 \mathrm{~m}$ above ordnance datum (aOD), just above the base of the site at approximately $1 \mathrm{~m}$ aOD (Figure 9). Details of the design, installation and operation of these wells are described by Beaven et al. $(2005 ; 2007)$ and Cox et al. (2006).
Three electrical imaging Wenner-alpha arrays, each with 50 electrodes at $5 \mathrm{~m}$ interelectrode spacing, were installed on the surface of the landfill (elevation of approximately $32 \mathrm{~m}$ aOD) perpendicular to the horizontal drains (Figure 10). The installation of the cables on the landfill involved excavating small trenches, approximately $30 \mathrm{~cm}$ deep, through an intermediate soil cap to the top surface of the waste, along which the imaging cables were laid. 

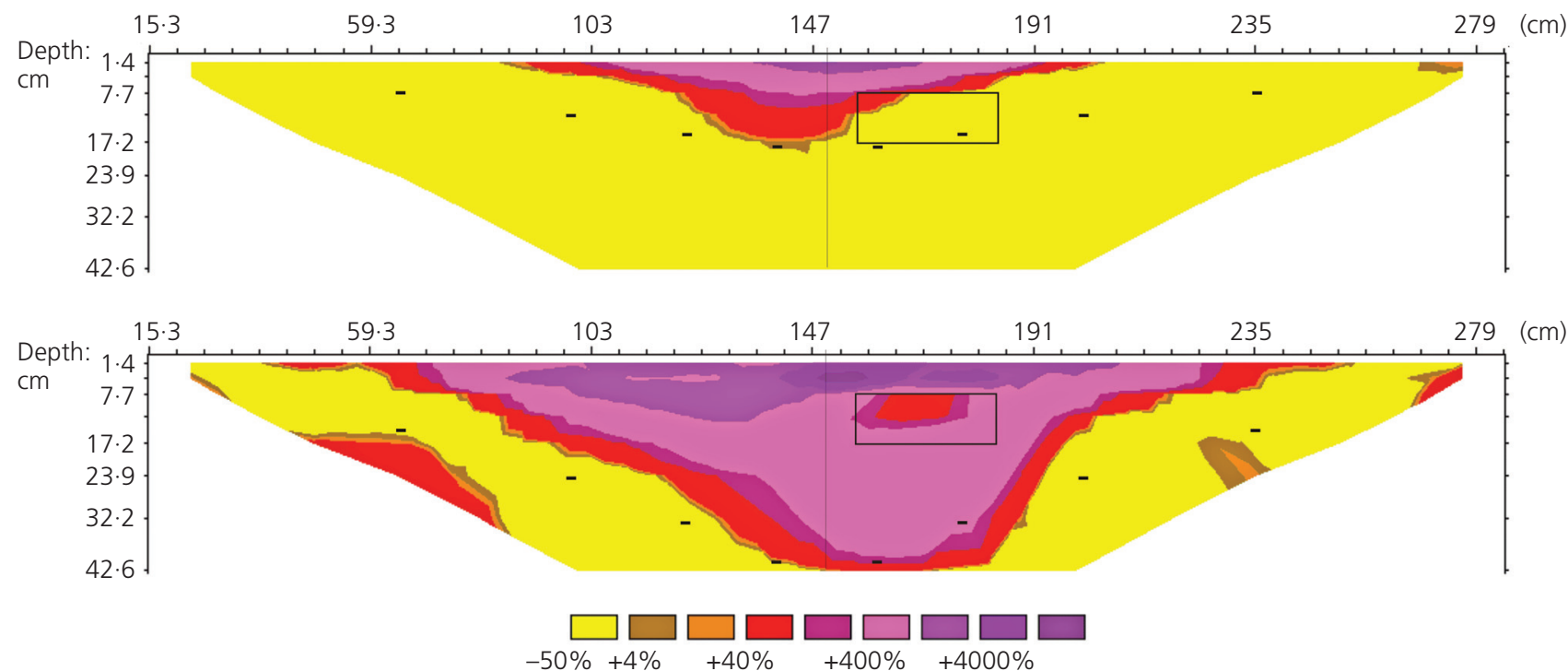

Figure 7. Percentage changes in resistivity recorded during a drainage experiment in a non-homogeneous medium. The heterogeneity is composed of absorbent paper and is much less freely draining than the surrounding sand. Its location is indicated by the rectangle in the right-hand section of the image. The water levels recorded in the observation wells are shown by the black dash marks. For colour versions of figures please see www.

icevirtuallibrary.com/content/article/10.1680/warm.2011.164.2.79
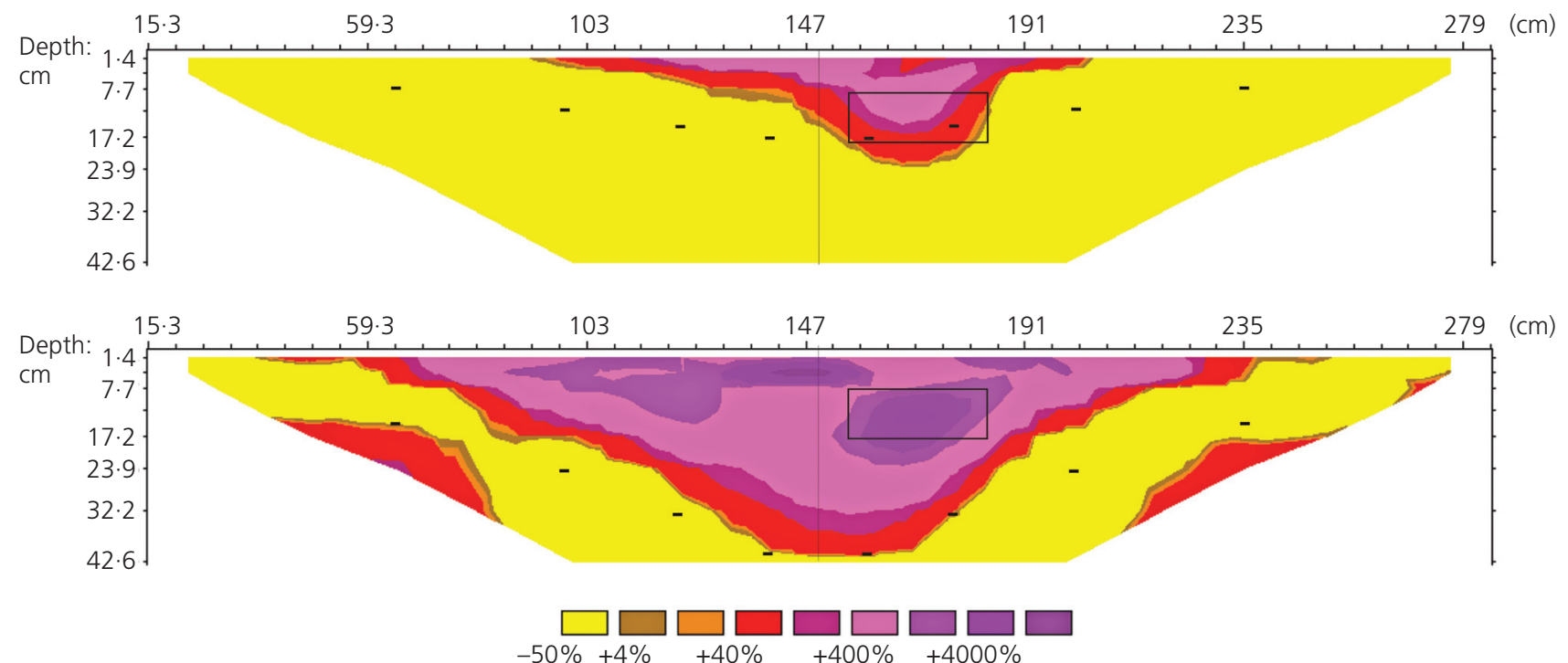

Figure 8. Percentage changes in resistivity recorded during a drainage experiment in a non-homogeneous medium. The heterogeneity is composed of gravel and is able to drain more freely than the surrounding sand. The location of the gravel is indicated by the rectangle in the right-hand section of the image. The water levels recorded in the observation wells are shown by the black dash marks. For colour versions of figures please see www.

icevirtuallibrary.com/content/article/10.1680/warm.2011.164.2.79 


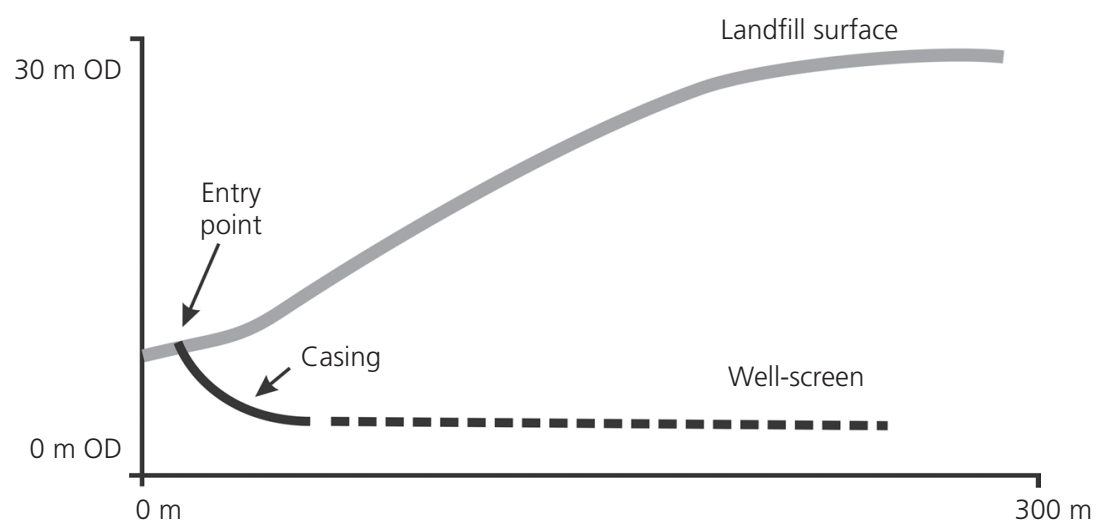

Figure 9. Typical profile of horizontal drain (OD = ordnance datum)

To prevent damage to the PVC insulation on the cables, the trenches were dressed with sand. Stainless steel electrodes were installed in small holes augered vertically into the base of the trench and backfilled with a wet sand and bentonite mixture to ensure and maintain a good electrical contact with the ground.

An assessment of the optimum direct current cycle for data gathering in this setting, including current strength and on and off times, are described in detail in Moore (2004). Tests were undertaken to determine the error in the measurement of potential difference recorded at signal strengths varying between 5 and $100 \mathrm{~mA}$. Although the higher currents increase the signal-to-noise ratio and reduce the error in the recorded measurements of potential difference, continuous use of the maximum $100 \mathrm{~mA}$ current output would have required vast battery supplies and could lead to shorter instrument life. The optimum applied current was chosen to be a combination of 10,20 and $50 \mathrm{~mA}$, with the larger currents being used at greater interelectrode spacings with an acceptable error on repeat readings of less than $2 \%$. Readings were collected using a square wave input function with a $1 \mathrm{~s}$ on time and $0.5 \mathrm{~s}$ off time. The polarity of applied current was reversed between readings.

The configuration of the horizontal drains meant that the drainage pattern and hence changes in resistivity were also likely to be predominantly two-dimensional, thus reducing the potential for out-of-section artefacts in the interpretation of the electrical imaging results. To provide comprehensive data on the dewatering and to aid the validation of the time-lapse electrical imaging, piezometers at multiple levels were installed in lines perpendicular to the horizontal drains (Figure 10). Leachate levels measured in April 2000 before dewatering recorded piezometric heads of up to $18 \mathrm{~m}$ aOD.

The results reported in this paper concentrate on data taken from resistivity line 1 that tracked the dewatering around HW1. Further details of the techniques applied and data from the whole survey are provided by Moore (2004) and Taylor and Barker (2003).

In the early stage of the drainage programme images were recorded on an approximately weekly basis, but this was reduced to monthly when the effects of the dewatering reduced, and it became apparent that changes in resistivity were not significant over the shorter timescale. Monitoring continued over the period July 2000 to January 2002, although the most interesting results occurred in the first 8 months.

\subsection{Review of the field results}

The electrical image recorded on line 1 before the start of dewatering is shown in Figure 11. This represents the baseline data against which the time-lapse images in Figure 12 are derived. Figure 13 also shows values of daily rainfall at the site over the first 8 months of the survey, and the volumes of leachate and landfill gas extracted from well HW1 during a four-step pumping test, in which the drawdown in the well was progressively increased in each step (Beaven et al., 2007).

The results of the time-lapse imaging showed there to be no general dewatering of the waste by the horizontal wells, a finding backed up by an independent piezometric monitoring programme (Beaven et al., 2005; 2007) that indicated that although there had been considerable reduction in pore pressure of a confined layer within the waste, there had been little or no desaturation (Figure 14).

An area of high resistivity developed directly above one of the horizontal wells soon after the start of pumping (Figure 12, $23 / 08 / 00$, symbol ' $a$ '). This is thought to be due to the release of gas within the leachate and a consequential reduction in the water-filled porosity in response to a pore water pressure reduction (caused by pumping). This correlates extremely well with the model predictions of Beaven et al. (2005). Over the 18-month monitoring period there was a net decrease in 


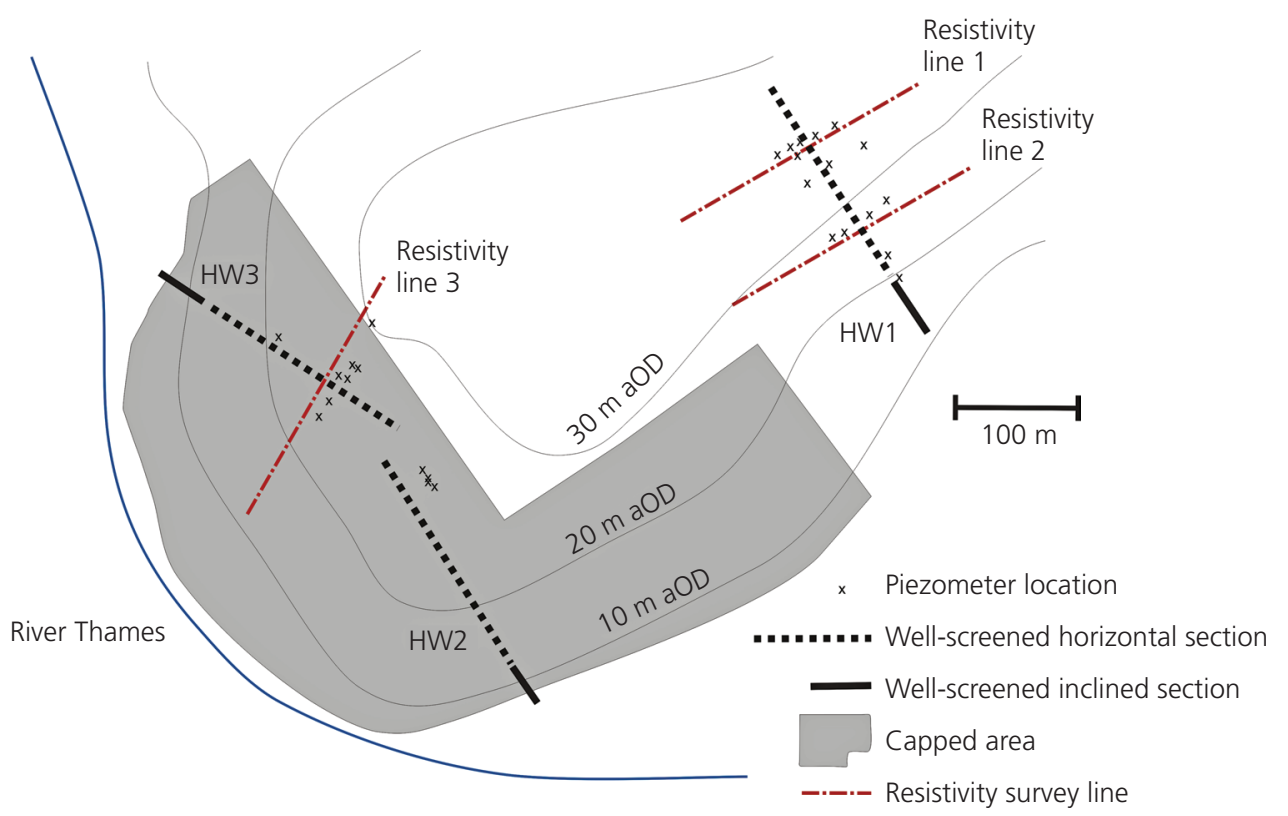

Figure 10. Location of horizontal dewatering wells, piezometers

resistivity within the landfill. Based on the relatively constant conductivity of the leachate extracted over that period and an assumed limited change in temperature within the waste material during the 18 months, the decrease in resistivity was attributable solely to an increase in the quantity of pore spaces filled with liquid and caused by exceptionally high rainfall over the monitoring period, as verified by rainfall data obtained from a local weather station.

The independent interpretation of the time-lapse electrical imaging and the observation well data both support a conceptual model of a saturated hydrogeological unit at depth, which is confined or semiconfined and which has experienced a reduction in hydraulic head as a result of the drainage regime but has not become desaturated. The electrical imaging has complemented the hydrogeological monitoring data and has identified processes (e.g. the formation and dissolution of gas into leachate above the well) that are supported by independent modelling results (Beaven et al., 2005; 2007), but not picked up by the monitoring.

The images in Figure 12 show changes in apparent resistivity over time that can be related to the location and known operation of the horizontal drain. However, it is important to develop an understanding of the extent to which certain features

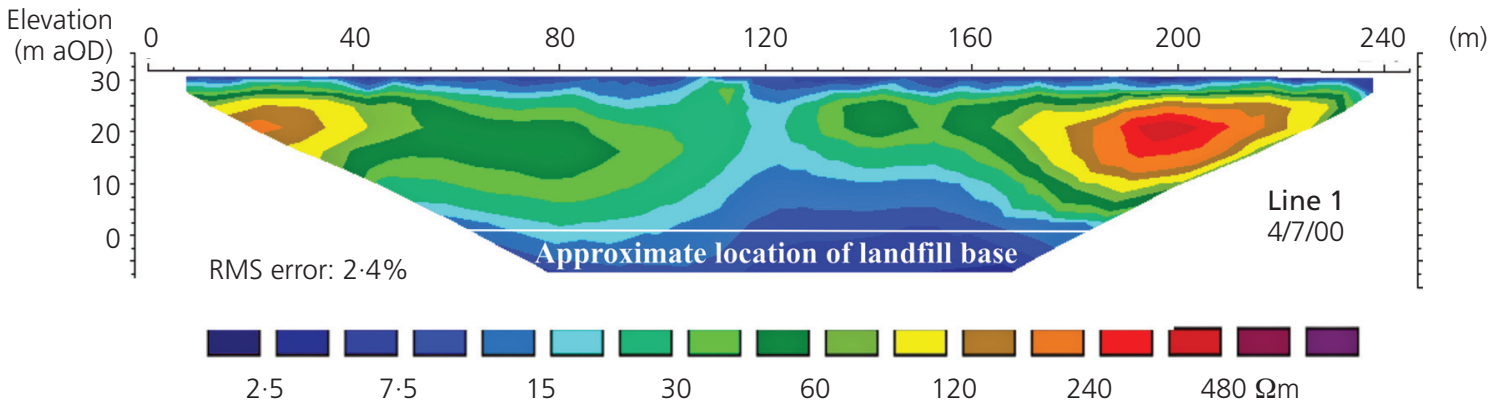

Figure 11. Electrical images from line 1 with contours of resistivity in Ohm-metres $(\Omega \mathrm{m})$. The images were recorded before the start of the drainage programme and were inverted with a fixed resistivity of $9 \Omega \mathrm{m}$ at the base of the landfill (aOD = above ordnance datum; $\mathrm{RMS}=$ root mean square). For colour versions of figures please see www.icevirtuallibrary.com/content/article/10.1680/ warm.2011.164.2.79 
Resolution of electrical imaging of fluid

movement in landfills

Jolly, Beaven and Barker
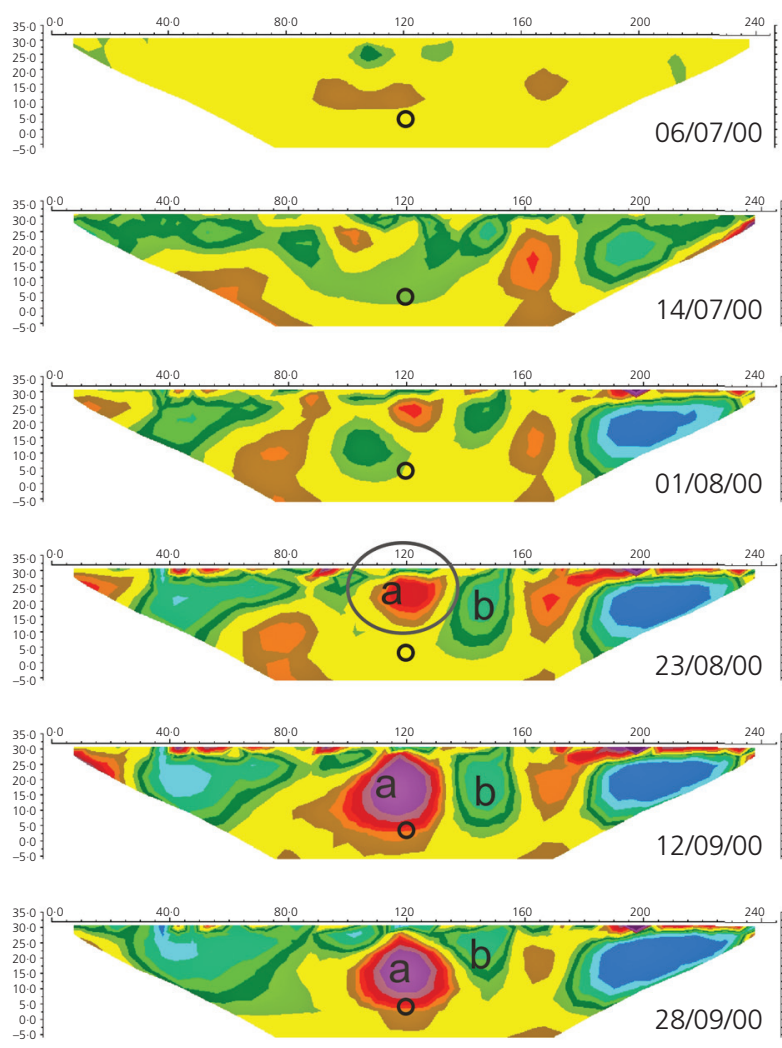
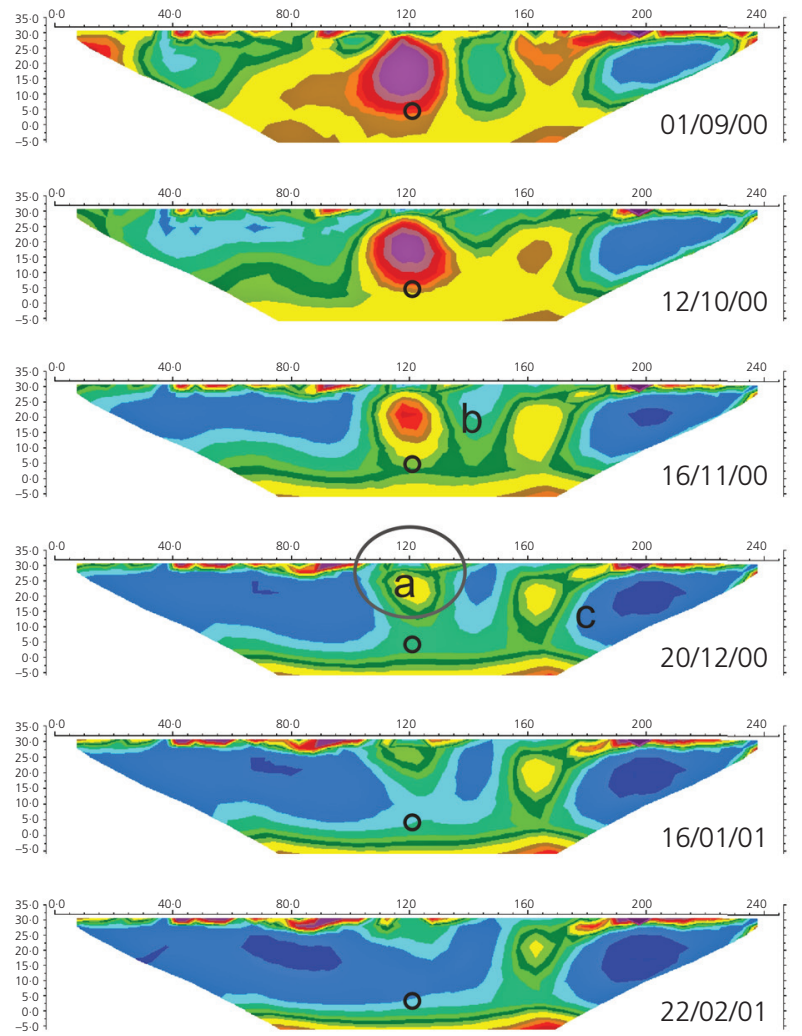

o Location of horizontal well

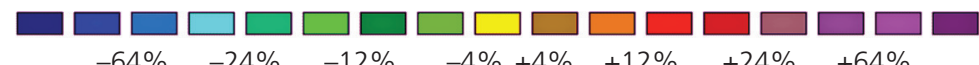

Figure 12. Sample of results from the time-lapse electrical imaging. Percentage change in resistivity recorded over the landfill, where a decrease in resistivity indicates an increase in moisture content and an increase in resistivity indicates a decrease in moisture content. For colour versions of figures please see www.icevirtuallibrary.com/ content/article/10.1680/warm.2011.164.2.79

on the images reflect true processes and also to identify which features may be artefacts of the data inversion process. As discussed above, independent evidence supports the notion that there is not wholesale dewatering at depth, but localised depressurisation resulting in the release of landfill gas into saturated waste above and around the horizontal drain. This is reflected on the inverted sections in Figure 12 as the development of a bulbous zone of higher resistivity (marked by symbol ' $a$ ' on sections) above the well. At its largest this anomaly measures at least $40 \mathrm{~m}$ across. On either side of this central feature (and especially to the right) the sections show the development of lower resistivity areas (b) which could be interpreted as an increase in water content, although (from the results of the laboratory study) are more likely to be artefacts. Other areas of high and low resistivity develop on the sections but from a preliminary assessment it is difficult to differentiate between real effects and artefacts.
Consequently, to provide added confidence in the electrical imaging results there needs to be better understanding of the spatial resolution of the technique, the scale of any anomaly within the waste material that can be realistically detected and the location and magnitude of inversion artefacts that are generated. To investigate these aspects a modelling exercise was carried out and is presented in Section 4.3.

\subsection{An assessment of the resolution}

For any electrical imaging survey, the interelectrode spacing implies certain constraints on the resolution. The magnitude of the contrast in resistivity between the anomaly being investigated and the surrounding 'host' media (or in the case of time-lapse imaging, the magnitude of the change in resistivity over time) is also important. In broad terms, we would not expect to resolve accurately the shape of features very much smaller than the electrode spacing, although the effect of a 

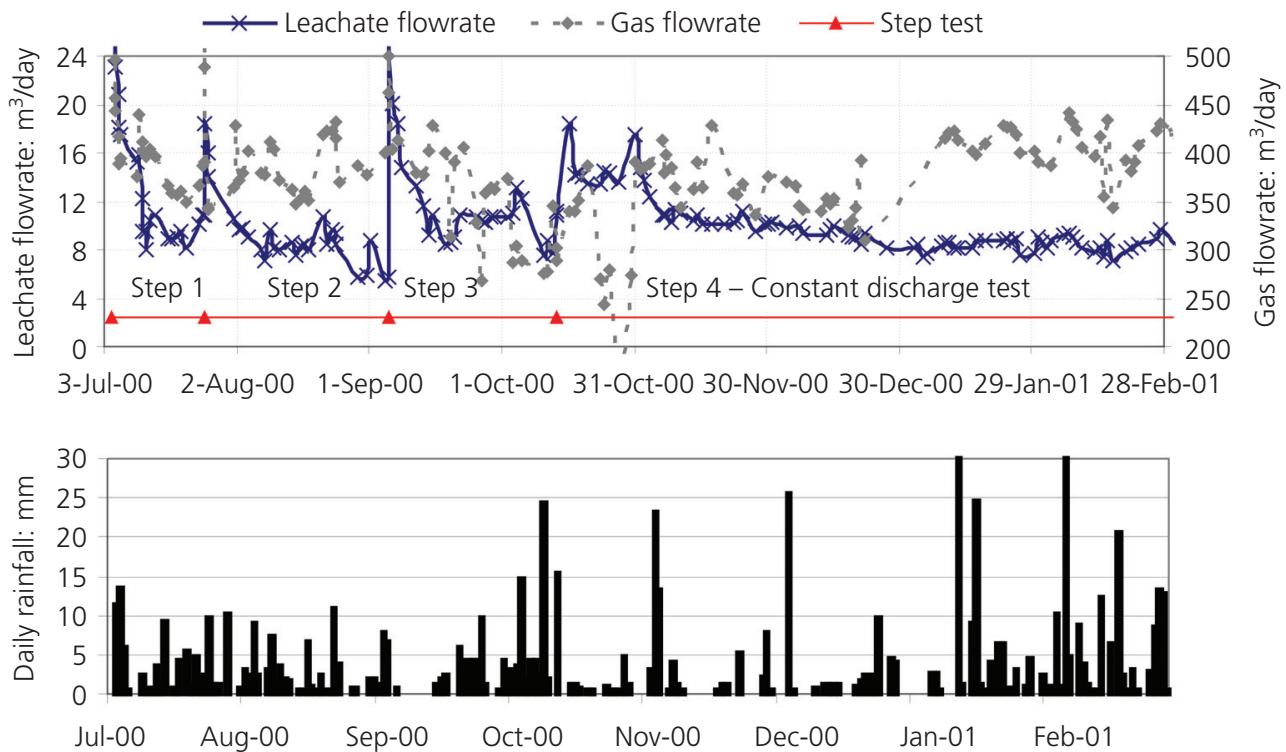

Figure 13. Volume of leachate and gas extracted from horizontal well HW1 and daily rainfall data

small localised event with a high contrast in resistivity (or a large change in resistivity over time for time-lapse imaging) within an element of this size would be likely to be detected.

For electrical imaging surveys carried out using electrodes at the ground surface, larger electrode spacings are required to pass current deeper into the ground to obtain measurements of resistivity at greater depths. An inevitable consequence of having to use larger electrode spacings is a decrease in the spatial resolution with depth. Very approximately, the depth of investigation is approximately half the electrode spacing and therefore a minimum electrode spacing of approximately $60 \mathrm{~m}$ is required to acquire information at a depth of $30 \mathrm{~m}$. At larger electrode spacings, and for a constant resistivity contrast,

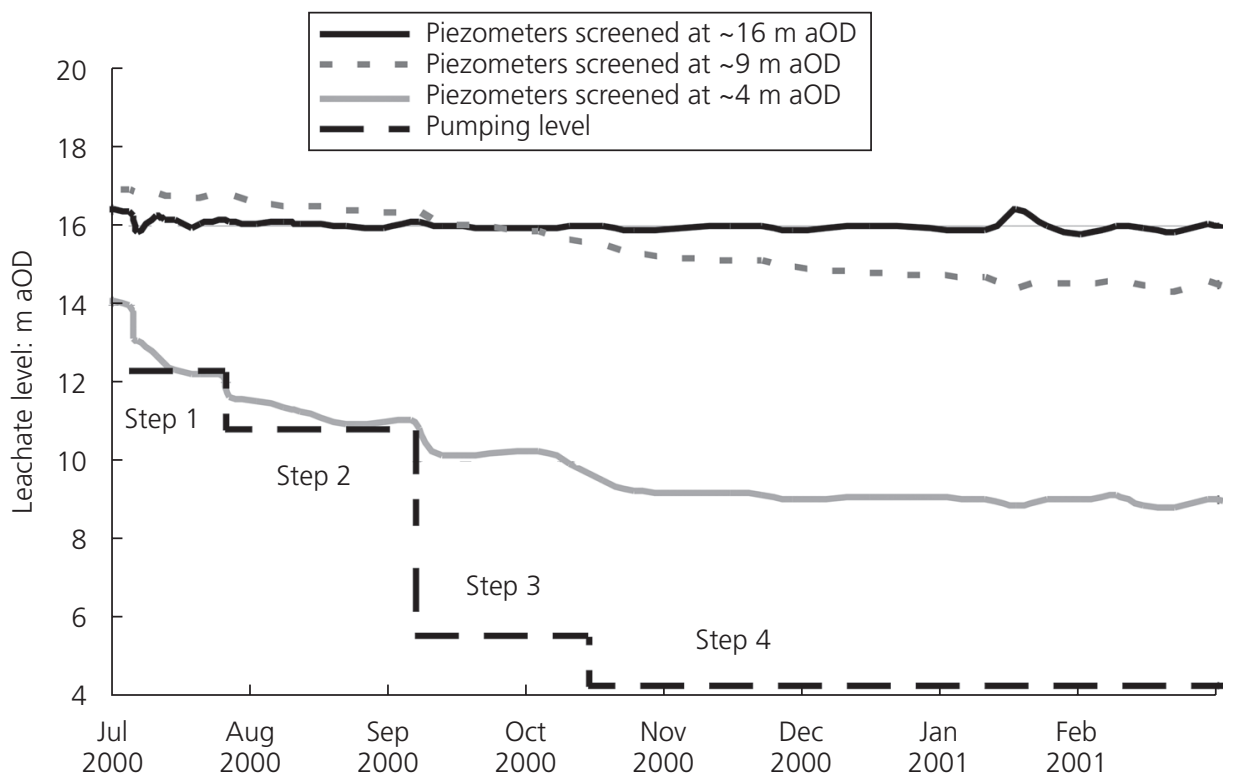

Figure 14. Typical piezometric response in discrete level piezometers around HW1 ( $\mathrm{aOD}=$ above ordnance datum) 
the minimum size of anomaly that can be detected also increases.

To investigate the spatial extent to which changes in resistivity must have occurred to produce the time-lapse images presented in Figure 12 and to demonstrate more clearly issues relating to image resolution, a modelling exercise has been carried out using the forward modelling program RES2DMOD (Loke, 1999). A simple two-layer model was constructed as the starting reference model, with an upper resistivity of $60 \Omega \mathrm{m}$ representing the waste material and a lower resistivity of $9 \Omega \mathrm{m}$ representing a basal clay layer and an interface at a depth of $31 \mathrm{~m}$. Figure 15 shows four different subsurface models, each assuming that infiltration (represented by a lower resistivity of $15 \Omega \mathrm{m}$ ) is occurring in discrete vertical channels of varying thicknesses, geometries and spacings. It is not suggested that these models necessarily accurately represent infiltration at a landfill, but their main purpose is to investigate resolution. The resulting time-lapse electrical image for each of these four infiltration patterns (the calculated percentage change in resistivity from the reference case with no channelling) is shown below each model, giving a direct comparison between a ground (or at least physical) truth and the calculated changes in resistivity.

Model 1 depicts five vertical infiltration 'channels' approximately $5 \mathrm{~m}$ wide spaced at $45-55 \mathrm{~m}$. The electrical imaging clearly identifies each of these channels, but instead of depicting sharp vertical features, rather bulbous distortions to the imaging field are observed with increasing depth. At the landfill surface, the channels are resolved to a width of approximately $10 \mathrm{~m}$, whereas at a depth of approximately $25 \mathrm{~m}$ the horizontal resolution is nearer $30-40 \mathrm{~m}$, approximately six to eight times their actual width. There are also areas of increased resistivity in between the infiltration channels on these images that are not real and are purely artefacts of the inversion process. The anomalous appearance of areas of increased resistivity is evident on all of the four models, although the extent of these anomalies is suppressed at depth as the resolution of the technique reduces. The magnitude of the artefacts seen in the models in Figure 15 appears to be proportional to the magnitude of the true change in resistivity. Many previous studies (Grellier et al., 2008; Moreau et al., 2004; Rosqvist et al., 2005) have studied the impact of leachate recirculation on apparent resistivity and have noted, in addition to zones of decreased resistivity directly around the leachate recirculation infrastructure, peripheral zones of increased resistivity. These zones have been interpreted as evidence of enhanced landfill gas flow or migration causing a reduction in water content. These conclusions have usually been drawn without discussing inversion artefacts as a possible cause.

In relation to the field results (Figure 12), the large positive anomaly that starts to develop on 23 August 2000 and continues until 20 December 2000 is not considered to be an artefact, as the magnitude of the increase in resistivity is far greater than the surrounding decrease in resistivity and is considered to be related to a true increase in resistivity. However, as discussed above, the spatial extent of the increase in resistivity is likely to be less well constrained at depth.

Model 2 keeps the five infiltration channels of model 1, but introduces various merging and bifurcations of the flow paths. Where two flow paths merge near the top of the model, this is picked up in the electrical imaging, but any 'structure' below approximately $10 \mathrm{~m}$ is not discernible.

Model 3 introduces a network of more closely spaced infiltration channels, at a spacing that varies between 5 and $20 \mathrm{~m}$. With this more closely spaced network, the imaging is unable to differentiate any structure at a depth below $10 \mathrm{~m}$, although the presence of artefacts penetrates to a deeper level. This infiltration model is beginning to represent the time-lapse images that were recorded in the field (Figure 12, 22/02/01). It is considered that model 3 represents the minimum frequency of infiltration channels that could give rise to the lateral extent of changes in resistivity observed in Figure 12. It is considered that model 3 is approaching the limit of lateral resolution of this electrical imaging technique, particularly at depth within the landfill. While a denser network of infiltration channels could be resolved at shallow depths, the course of the channels at depths greater than approximately $10 \mathrm{~m}$ could not be resolved. Therefore, although it cannot be stated that model 3 represents the actual infiltration pattern that occurred during the field trial, it does provide a useful understanding of plausible flow mechanisms and quantifies the sensitivity of this electrical imaging technique.

It is noted that the depth to which changes in resistivity have occurred is well represented within the time-lapse image calculated for model 3 . To test the converse of this, when the modelled infiltration extends to only $15 \mathrm{~m}$ depth within the waste (model 4), the time-lapse electrical image does not show large changes in resistivity below this depth.

It has been demonstrated by this modelling exercise that the resolution of individual infiltration channels reduces as the separation between them is reduced and that this effect is exacerbated with depth (with increased electrode spacing), but that the depth of infiltration of the channels is well constrained. That is, the technique has good vertical resolution but changes in electrical properties in the horizontal direction are less well constrained. There is a minimum lateral separation of infiltration channels that can be resolved by this electrical imaging technique that depends on the depth of investigation (which is directly related to the electrode spacing for surface electrode surveys) and the magnitude of the anomaly. 
Waste and Resource Management

Volume 164 Issue WR2
Resolution of electrical imaging of fluid

movement in landfills

Jolly, Beaven and Barker
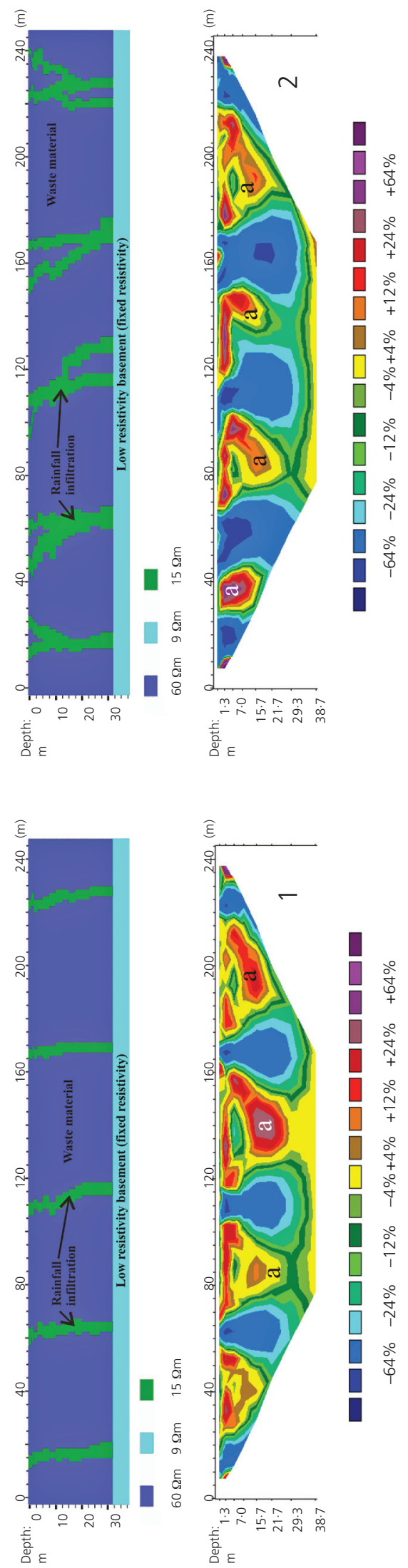
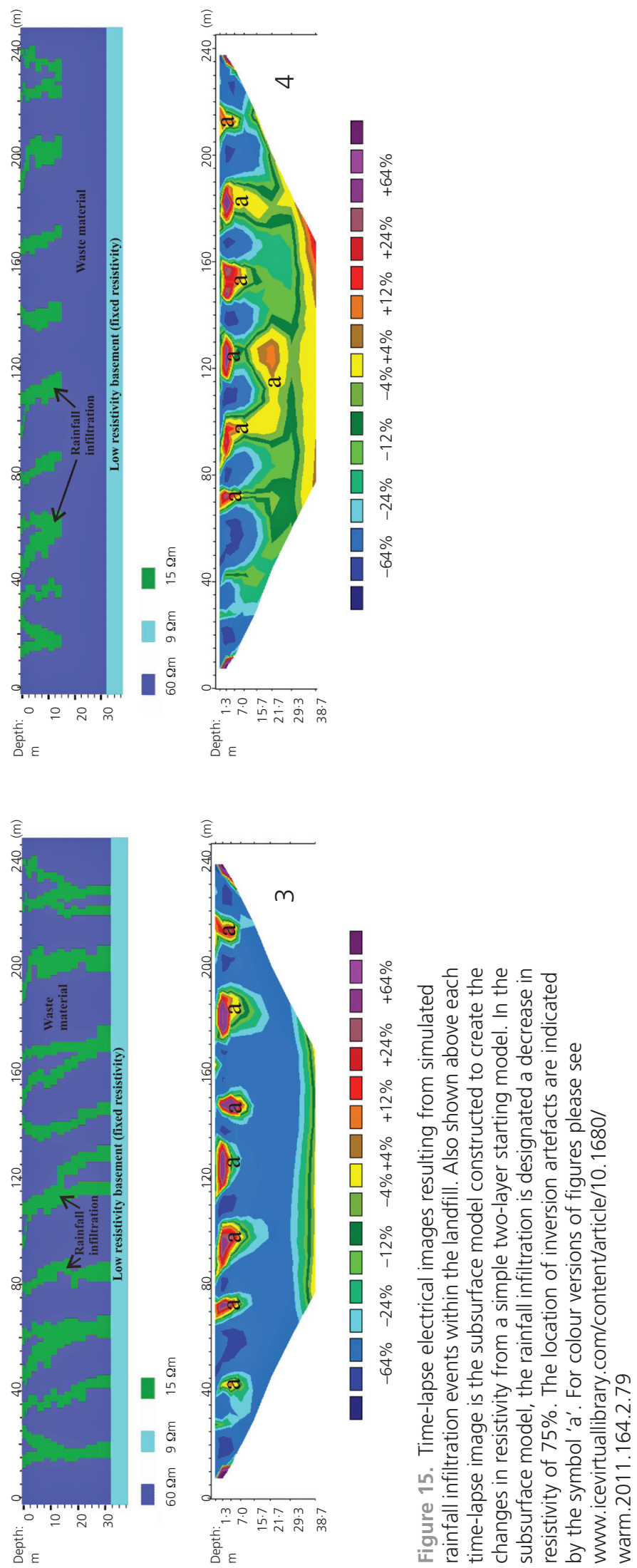


\section{Discussion}

Interpretation of the results shown in Figure 12 in the context of both the laboratory and modelling studies enables the practitioner to make a more informed assessment of the anomalies recorded in the field trial.

The laboratory dewatering study provides direct physical evidence that resistivity surveying can detect temporal and spatial variations in the capillary fringe and in the water content in and around hydraulic inhomogeneities that are not identified using conventional monitoring techniques (e.g. piezometers). In addition, the laboratory study clearly demonstrated the resolution of the resistivity at different points along the section (resolution reduced towards the ends and with depth), and also the creation of inversion artefacts that mirrored real causative effects. The laboratory study, by comparison, also provides added confidence to the study of artefacts using forward modelling techniques.

In the modelling study, any observed artefacts were always of a lesser magnitude than the change in resistivity arising from the causative body. Consequently, we can be confident that in this case the increase in resistivity that develops strongly above the horizontal well from late August through to October is a real effect and not an artefact of the inversion process. This conclusion can be drawn independently from the other supporting evidence provided by Beaven et al. (2005) and described in Section 4.2. The modelling also shows that the resolution of the technique decreases with depth and therefore we can also be sure that the causative body will be somewhat smaller than the size of anomaly reported. However, without knowing more information about the actual resistivity increase that occurred (in comparison with initial resistivities) it is difficult to determine the true size of the causative body.

The areas of decreased resistivity immediately adjacent to the large increase in resistivity cannot be interpreted confidently as a real decrease in resistivity. This alternating pattern is similar to the artefacts noted in the modelling study, and therefore guides the interpreter to place less confidence in these areas of the cross-section.

The large areas of decreased resistivity in the upper left side of the sections, which started developing in July 2000 and became very pronounced from November onwards, can be confidently interpreted as a real increase in resistivity. The laboratory modelling study clearly demonstrated the ability of the technique to delineate large changes of resistivity in the subsurface accurately. However, the ability to resolve the spatial extent of changes in resistivity does decrease with depth; therefore the confidence in the interpretation also decreases with depth.
In physical terms the likely causes for the decrease in resistivity could either be from rainwater infiltration and/or from a general increase in landfill temperatures (e.g. Grellier et al., 2006). There is physical evidence of high rainfall over this time, but there was no temperature monitoring of the landfill. Hanson et al. (2010) have indicated that significant temperature changes can occur in landfills over the timescale of this experiment, but there is no way of 'knowing if this occurred.

Taking rainfall infiltration as a cause, then the resolution study helps with interpretation. If rainfall infiltration was uniformly distributed then there should be a uniform decrease in resistivity from the surface downwards. The actual results show that there are perturbations to the resistivity field in the upper 3 or $4 \mathrm{~m}$ but below $5 \mathrm{~m}$ variations are smoothed out. The modelling study showed that spatially distributed infiltration in channels at a spacing of approximately $5 \mathrm{~m}$ gave a similar response, although the effects of individual channels are resolved to a depth of nearer $10 \mathrm{~m}$. With this knowledge, we can confidently interpret that the decrease in resistivity recorded on the landfill during the winter months is a real change. Based on our knowledge of waste material, it is unlikely that water is infiltrating the landfill as a uniform front and is occurring within discrete flow paths but at a scale of less than $5 \mathrm{~m}$.

The modelling also shows that the water is likely to be penetrating to a reasonable depth within the waste material. This is perhaps a surprising conclusion given our understanding of the hydraulic properties of waste material, but it is an interpretation well supported by the modelling study.

\section{Conclusions}

There are a number of significant conclusions that can be drawn from this work.

(a) The field scale validation of the interpretation of the time-lapse images against observation well data, in terms of the lack of desaturation, and against the rainfall data, in terms of an overall decrease in resistivity in response to heavy rainfall, provides great confidence in the use of electrical imaging for the monitoring of leachate conditions and net changes in moisture content within a landfill. However, no data were available to constrain the temperature variation within the waste material and it is suggested that any future time-lapse imaging surveys carried out at landfill sites should include the monitoring of temperature variation, both spatially and temporally, within the waste material.

(b) Time-lapse electrical imaging detected the infiltration of rainwater through the surface of the landfill, the timing and location of which could not have been detected by any other monitoring technique. Therefore, time-lapse 
imaging has enormous potential for the long-term monitoring of the effects of landfill cap integrity.

(c) The appearance of anomalous changes in resistivity and reduction in resolution at depth in both the laboratory and model time-lapse images emphasise the importance of fully understanding the limitations of any monitoring technique. The merits of time-lapse electrical imaging in the monitoring of landfill processes are indisputable, but as with other more common methods of monitoring fluid in the subsurface, such as boreholes and piezometers, ambiguity in the interpretation exists and it is the responsibility of the skilled practitioner to make robust interpretations of the data.

\subsection{Advantages and limitations of the technique}

Electrical imaging has many advantages in the field setting, as it is inexpensive, non-invasive and spatially extensive in its measurement.

\subsubsection{Complementary methods}

The results of the laboratory-based study indicate that timelapse electrical imaging can be used to complement standard hydrogeological measurement techniques and can detect and locate contrasts in hydraulic properties in a heterogeneous subsurface not readily identifiable by point measurement techniques. This is a very important result, and could prove to be an invaluable addition to conventional hydrogeological studies.

The combined use of time-lapse electrical imaging with hydrogeological monitoring of a field scale dewatering trial resulted in mutually validating interpretations of the separate datasets and therefore a better constrained conceptual model of the hydrogeological conditions within the landfill.

\subsubsection{Artefacts in the results}

Both the laboratory experiment that showed a direct comparison between physical conditions and inverted geophysical sections and the forward modelling exercise highlighted the problem of artefacts created during the inversion of apparent resistivity data. Many previous resistivity studies applied to landfills have not taken adequate account of this factor. The dominant factors contributing to anomalies were the magnitude of the resistivity contrasts and the location of the anomaly. Therefore, it is recommended that a short modelling exercise be carried out in conjunction with the interpretation of timelapse electrical imaging results, to establish the magnitude and location of any spurious anomaly that may arise. This should enable a more comprehensive understanding of all the features within the time-lapse image.

\subsubsection{Resolution}

Caution must be exercised in the interpretation of electrical imaging, particularly at depth as resolution decreases. The technique is able to detect the impact of discrete events that change the resistivity of the subsurface, but is not necessarily able to resolve the exact location or shape of what is causing the change. As an example, the imaging technique was not able to differentiate the modelled presence of discrete infiltration channels at a separation of between 5 and $20 \mathrm{~m}$ below a depth of $10 \mathrm{~m}$.

\section{Acknowledgements}

The authors wish to thank Veolia Environmental Ltd. for their support in the use of their site and the project sponsors Engineering and Physical Sciences Research Council (EPSRC).

\section{REFERENCES}

Beaven RP, White JK and Lucas P (2005) Modelling the performance of a horizontal leachate drain. Proceedings of the 10th International Waste Management and Landfill Symposium, Cagliari.

Beaven RP, Powrie W and Cox SE (2007) Operation and performance of horizontal wells for leachate control in a waste landfill. Journal of Geotechnical and Geoenvironmental Engineering 133(8): 1040-1047.

Benson CH, Barlaz MA, Lane DT and Rawe JM (2007) Practice review of five bioreactor/recirculation landfills. Waste Management 27(1): 13-29.

Clement R, Descloitres M, Gunther T, et al. (2010) Improvement of electrical resistivity tomography for leachate injection monitoring. Waste Management 30(3): 452-464.

Cox SE, Beaven RP, Powrie W and Cole DJ (2006) Installation of horizontal wells in landfilled waste using directional drilling. Journal of Geotechnical and Geoenvironmental Engineering 132(7): 869-878.

Environment Agency (2009) A Technical Assessment of Leachate Recirculation EA R\&D Report: SC030144/R1. See http://publications.environment-agency.gov.uk/pdf/ SCHO1109BRJC-e-e.pdf (accessed 25/02/11).

Grellier S, Robain H, Bellier G and Skhiri N (2006) Influence of temperature on the electrical conductivity of leachate from municipal solid waste. Journal of Hazardous Materials 137(1): 612-617.

Grellier S, Guerin R, Robain H, et al. (2008) Monitoring of leachate recirculation in a bioreactor landfill by 2-D electrical resistivity imaging. Journal of Environmental and Engineering Geophysics 13(4): 351-359.

Guerin R, Munoz ML, Aran C, et al. (2004) Leachate recirculation: moisture content assessment by means of a geophysical technique. Waste Management 24(8): 785-794.

Hanson JL, Yesiller N and Oettle NK (2010) Spatial and temporal temperature distributions in municipal solid waste landfills. Journal of Environmental Engineering 136(8): 804-814.

Jolly J, Barker R, Beaven RP and Herbert A (2007) Time-lapse electrical imaging to study fluid movement within a landfill. 
Proceedings of the 11th International Waste Management and Landfill Symposium, Cagliari.

Knox K, Beaven RP, Rosevear A and Braithwaite P (2007) A technical review of leachate recirculation. Proceedings of the 11th International Waste Management and Landfill Symposium, Cagliari (CD only).

Loke MH (1999) RES2DMOD version 2.2. Rapid 2D resistivity forward modelling using the finite-difference and finite element methods: copy written freeware. M.H. Loke, 22 pp.

Loke MH and Barker RD (1996) Rapid least-squares inversion of apparent resistivity pseudosections using a quasi-Newton method. Geophysical Prospecting 44(1): 131-152.

Moore JM (now Jolly) (2004) Fluid Flow Characterisation Using Time-Lapse Electrical Imaging. PhD thesis, University of Birmingham.

Moreau S, Bouyè J-M, Duquennoi C, Barina G and Oberti O (2004) Electrical resistivity survey to investigate biogas migration under leachate recirculation events. Proceedings of Waste 2004; Integrated Waste Management and Pollution Control Policy and Practice, Research and Solutions, Stratford-upon-Avon.

Olayinka Al and Yaramanci U (2000) Assessment of the reliability of $2 \mathrm{D}$ inversion of apparent resistivity data. Geophysical Prospecting 48(2): 293-316.

Park S (1998) Fluid migration in the vadose zone from 3-D inversion of resistivity monitoring data. Geophysics 63(I): $41-51$.

Rosqvist H, Dahlin T, Fourie A, et al. (2003) Mapping of leachate plumes at two landfill sites in South Africa using geoelectrical imaging techniques. Proceedings of the 9th International Waste Management and Landfill Symposium, Cagliari.
Rosqvist H, Dahlin T and Lindhé C (2005) Investigation of water flow in a bioreactor landfill using geoelectrical imaging techniques. Proceedings of the 10th International Waste Management and Landfill Symposium, Cagliari.

Rosqvist H, Dahlin T, Linders F and Meijer J-E (2007)

Detection of water and gasmigration in a bioreactor landfill using geoelectrical imaging and a tracer test. Proceedings of the 11th International Waste Management and Landfill Symposium, Cagliari.

Rosqvist H, Leroux V, Dahlin T, et al. (2011) Mapping landfill gas migration using resistivity monitoring. Waste and Rescource Management 164(1): 3-15, doi: 10.1680/ warm.2011.164.1.3.

Taylor S and Barker R (2003) Rainham Geophysical Monitoring - Application of Electrical Imaging to Landfills: Supplementary Monitoring. Final report RWNF09197 to SuNRISE Environmental Body. School of Geography, Earth and Environmental Sciences, University of Birmingham.

Taylor SB, White CC and Barker RD (1999) A comparison of portable and permanent landfill liner leak-detection systems. In Field Instrumentation for Soil and Rock ASTM STP1358 (Durham GN and Marr WA (eds)). American Society for Testing and Materials, West Conshohocken, PA, USA, pp. 157-174.

White JK, Beaven RP, Powrie W and Knox K (2011) Leachate recirculation in a landfill: Some insights obtained from the development of a simple 1-D model. Waste Management, doi: $10.1016 /$ j.wasman.2010.10.022.
WHAT DO YOU THINK?

To discuss this paper, please email up to 500 words to the editor at journals@ice.org.uk. Your contribution will be forwarded to the author(s) for a reply and, if considered appropriate by the editorial panel, will be published as a discussion in a future issue of the journal.

Proceedings journals rely entirely on contributions sent in by civil engineering professionals, academics and students. Papers should be $2000-5000$ words long (briefing papers should be 1000-2000 words long), with adequate illustrations and references. You can submit your paper online via www.icevirtuallibrary.com/content/journals, where you will also find detailed author guidelines. 